Agriculture suisse et mondialisation

\title{
Perspectives alternatives sur l'accès aux marchés et sur les exportations agricoles des pays du Sud
}

\section{Anna Crole-Rees}

\section{(2) OpenEdition}

12 Journals

Édition électronique

URL : http://journals.openedition.org/aspd/923

DOI : 10.4000/aspd.923

ISSN : 1663-9669

Éditeur

Institut de hautes études internationales et du développement

\section{Édition imprimée}

Date de publication : 1 avril 2002

Pagination : 39-48

ISSN : 1660-5934

\section{Référence électronique}

Anna Crole-Rees, «Perspectives alternatives sur l'accès aux marchés et sur les exportations agricoles des pays du Sud ", Annuaire suisse de politique de développement [En ligne], 21 | 2002, mis en ligne le 06 septembre 2012, consulté le 08 septembre 2020. URL : http://journals.openedition.org/aspd/923 ;

DOI : https://doi.org/10.4000/aspd.923 


\title{
PERSPECTIVES ALTERNATIVES SUR L'ACCÈS AUX MARCHÉS ET SUR LES EXPORTATIONS AGRICOLES DES PAYS DU SUD
}

\author{
Anna Crole-Rees*
}

\section{INTRODUCTION}

Le dernier rapport sur le développement mondial de la Banque mondiale (World Bank 2001) aborde la lutte contre la pauvreté et la réduction de l'inégalité. Il préconise un meilleur accès à l'emploi et au revenu, cela particulièrement pour les plus défavorisés. L'accès à des marchés plus vastes et plus riches, c'est-à-dire aux marchés des pays à hauts revenus, est l'une des solutions promues par la communauté internationale (World Bank 2001).

La production et le commerce des biens et services se sont profondément modifiés durant les dernières décennies suite aux progrès dans le transport, à l'augmentation de la productivité, à l'évolution des goûts et des préoccupations des consommateurs dans les pays du Nord et à l'évolution des conditions-cadres dans les différents pays, au Nord comme au Sud.

Cette contribution se propose de présenter quelques aspects de la promotion des exportations agricoles des pays du Sud vers la Suisse, en prêtant une attention particulière aux opportunités et aux contraintes des labels dits «durables». La première partie passe en revue les principaux défis actuels que représente l'augmentation des exportations des produits des pays du Sud. La deuxième partie décrit les labels biologiques, les labels écologiques et ceux établis dans les pays du Sud. Enfin, la dernière partie propose quelques mesures pour accroître les exportations des produits provenant des pays du Sud.

\section{LES DÉFIS ACTUELS POSÉS AUX PAYS DU SUD POUR UN MEILLEUR ACCĖS À LA COMMERCIALISATION}

«Les Suisses sont des consommateurs exigeants et gâtés » ${ }^{1}$ (CBI et SIPPO 1999) : c'est ainsi que les consommateurs suisses (et des pays industrialisés en général) sont décrits dans une brochure destinée aux exportateurs vers la Suisse. Les habitudes de consommation ont fortement évolué durant les dernières décennies. Le consommateur dépense actuellement moins de $15 \%$ de son salaire pour son alimentation. Son goût pour l'exotisme a fortement augmenté, suite entre autres à ses voyages et à l'amélioration des moyens de transport des produits. Face à un nombre beaucoup plus important de produits issus de pays et de modes de production et de transformation différents, face à une variété de labels et de marques, le

* Consultante, économie/développement, av. d'Echallens 13, 1004 Lausanne, tél. 021/625 64 64, e-mail $<$ Anna_CroleRees@compuserve.com>. L'auteure remercie Olivier Matringe pour sa lecture critique et ses nombreux et passionnants commentaires.

1. Traduit de l'anglais «Swiss people are spoiled and demanding consumers». 
client est plus difficile à fidéliser et ses choix plus volatiles. Il aime la nouveauté, mais peut être également fidèle à ses produits préférés. Il se montre très exigeant quant à la qualité et à la fraîcheur des produits, mais aussi quant à la commodité des produits, allouant de moins en moins de temps à la préparation des repas et aux achats. La régularité de l'approvisionnement, c'est-à-dire la possibilité de retrouver les mêmes fruits ou légumes tout au long de l'année et de choisir et diversifier son alimentation, constitue un critère de choix pour le consommateur. En outre, suite à l'amélioration des moyens de communication, il est de plus en plus préoccupé par des questions d'ordre social et environnemental.

Les circuits de distribution et la demande des importateurs se sont aussi profondément modifiés pour répondre aux changements de consommation et pour contrer une demande quantitative globale au Nord relativement stagnante. Du côté de la distribution, les tendances actuelles sont à une forte concentration et consolidation. De nos jours, $50 \%$ à $75 \%$ des ventes de fruits en Europe se font dans les supermarchés (Rabobank 2001). Les étalages présentent des produits de plus en plus hétérogènes. Pour chaque type de produits, le consommateur peut choisir entre des emballages de grandeurs variables ou opter pour la vente libre, on lui propose des niveaux de transformation et des marques différents, des produits avec ou sans additifs tels qu'agents conservateurs ou éléments nutritifs. De leur côté, les importateurs élargissent leurs services et se réorganisent. La longueur des circuits d'importation est réduite afin de diminuer les coûts. De nouvelles activités telles que l'emballage sont intégrées. Ils doivent aussi travailler avec des quantités plus importantes afin d'approvisionner des distributeurs de plus en plus grands. C'est pourquoi ils recherchent un nombre de fournisseurs optimal, leur permettant de répartir les risques, de disposer de produits de qualité durant toute l'année et de minimiser les coûts de transaction. Un autre changement important concerne le rôle des normes et des labels. De facteurs d'harmonisation et de standardisation des produits, ils sont devenus des outils de marketing (Reardon et al. 2001). Une des conséquences est l'adaptation constante des exigences des différents types de labels et normes pour maintenir les parts de marché.

Les facteurs de valeur ajoutée comme la diversité, la commodité de présentation et d'achat, l'exclusivité et la qualité se retrouvent essentiellement au niveau de la vente au détail, autrement dit dans les étalages des pays du Nord. La compétition entre le Sud et le Nord pour l'acquisition de la valeur ajoutée est forte. Actuellement, les dernières phases d'apprêtage se font en général sur le lieu de consommation. L'emballage, pour des raisons de coûts de transport et de marque, est fait dans les pays industrialisés, obligeant les producteurs à exporter leur production en vrac, à moindre valeur ajoutée. Les efforts de semi-industrialisation, voire d'industrialisation, dans le domaine agricole sont multiples, mais rencontrent beaucoup de difficultés. La demande locale est en règle générale très limitée pour des produits nouveaux et/ou à forte valeur ajoutée du fait de revenus moyens, sinon très bas, cela malgré des opportunités croissantes dues au tourisme et au changement alimentaire dans les villes.

Les incohérences des politiques entre les marchés internationaux et locaux peuvent sérieusement entraver les opportunités de commercialisation et de transformation. La transformation locale peut être concurrencée par l'importation de produits à moindre prix. Citons par exemple l'importation de fripes d'Europe en Afrique de l'Ouest, freinant sévèrement le développement de l'industrie textile 
locale. Un autre exemple est le prix du blé importé d'Europe, inférieur à celui des céréales locales comme le mil et le sorgho. Il inhibe les efforts d'industrialisation locale et décourage les recherches en vue de nouveaux produits à base de ces céréales locales et pour concevoir des équipements adaptés (Diopp 1999). La progressivité et les prix tarifaires demeurent une barrière importante, limitant les efforts de transformation sur place. La Banque mondiale (World Bank 2001) rappelle que les tarifs à l'importation appliqués dans un certain nombre de pays industrialisés sont beaucoup plus élevés pour les produits transformés que pour les produits primaires. Notons cependant que l'Union européenne a maintenant ouvert son marché aux pays les moins avancés, levant toutes les barrières tarifaires et quotas à l'exception des armes depuis février 2001 et d'une période transitoire pour certains produits (riz, bananes et sucre). Espérons que les autres grands pays industrialisé adopteront de telles mesures rétablissant une certaine neutralité commerciale. Implicitement, c'est la disparité des ressources pour le soutien à la production et la transformation, soutien direct ou indirect, entre le Nord et le Sud qui est mise en exergue. En moyenne, les agriculteurs de l'Union européenne, du Japon et des Etats-Unis ont reçu 20'000 USD d'aide en 1998 et les subventions représentaient 40\% de leurs revenus bruts en 1999 (CNUCED 2001) alors que le secteur agricole de la plupart des pays d'Afrique a connu un démantèlement des soutiens suite aux libéralisations et privatisations des différents services en amont et en aval de la production. De plus, les réductions, voire suppressions d'obstacles aux importations de produits agricoles dans les pays du Sud augmentent la concurrence de la production locale avec les produits importés.

Les coûts de production et de transaction peuvent anéantir les efforts de commercialisation, rendant le produit non compétitif sur le marché mondial. Les coûts de transaction sont souvent plus élevés dans les pays du Sud qu'au Nord. Dans la filière des fruits et légumes, on considère qu'ils représentent $60 \%$ de la valeur finale (Rabobank 2001). Par exemple, les coûts de transport à l'intérieur de l'Afrique et vers l'Europe sont particulièrement importants; le taux de fret pour un conteneur de 40 pieds est de 6300 USD entre le Kenya et l'Europe alors qu'il coûte 4000 USD depuis 1'Argentine (Lhéraut 2001). La politique locale de transport et les facteurs d'offre et de demande influencent les coûts. Les tonnages exportables par avion depuis l'Afrique de l'Ouest sont dispersés entre une dizaine de pays principaux et l'ensemble constitue un volume estimé à 22'000 tonnes par année, soit le tiers de ce que le Kenya peut exporter à partir d'un seul aéroport (Lhéraut 2001).

Produire plus peut signifier l'ouverture vers d'autres marchés, et diverses expériences ont montré que l'augmentation physique de la production est possible. Toutefois, il existe plusieurs contraintes du fait que les exigences d'organisation et de marché croissent en conséquence et peuvent devenir des goulets d'étranglement. Les contraintes souvent citées sont relatives à la mobilisation des fonds, à l'absence de crédit, à la logistique, à l'insuffisance en gestion, aux problèmes de délai. Une difficulté considérée comme insoluble par certains exportateurs est le respect des normes alimentaires, surtout par les petits producteurs (FIDA 2001). 


\section{$\square$ Le label biologique}

Les labels biologiques apparaissent dès le début des années 1950 (Damary 2001). Leur objectif est de promouvoir une production selon des procédés respectant l'environnement et de commercialiser des produits bons pour la santé, sans résidus chimiques (Damary 2001). Actuellement, le consommateur suisse peut se nourrir entièrement de produits biologiques s'il est prêt à payer le surplus de prix pour obtenir de tels produits. Le total des ventes biologiques de détail d'alimentation et de boissons représente $2 \%$ du total en Suisse, dont près de $60 \%$ est vendu en supermarchés (ITC 1999, cité par FiBL et SIPPO 2001). Les perspectives à court et moyen terme pour les produits alimentaires d'origine biologique sont estimées de moyennes à bonnes selon le type de produits, soit une croissance de 10 et $30 \%$ environ (FiBL et SIPPO 2001).

Si certains distributeurs s'identifient aux principes de l'agriculture biologique, la plupart reconnaissent leur intérêt économique de par ses facteurs inhérents de valeur ajoutée: élargissement de l'offre de produits, image auprès des consommateurs, prime, etc. La vente de produits dits «biologiques » en Suisse comme en Europe n'est possible que si ces produits répondent à des critères de base définis par des normes étatiques. Comme Damary (2001) le souligne, il est toutefois préférable de mettre sur le marché un produit qui ait été certifié par un label privé. $\mathrm{Si}$ le producteur suisse peut choisir entre plusieurs labels, le producteur du Sud connaît de plus grandes difficultés d'accès à la certification. La première contrainte est l'asymétrie de l'information. Beaucoup de producteurs n'ont pas connaissance des obligations et des opportunités, bien que plusieurs de leurs produits soient cultivés de manière biologique de facto. L'absence, dans la majorité des pays du Sud, d'organe de certification locale reconnue par le pays importateur et donc le fait de devoir faire appel à des organismes à l'étranger, en règle générale situés dans les pays du Nord, augmentent considérablement les coûts. Enfin, le coût de certification (visites, formation pour la conversion, frais d'administration et contrôles) est élevé. En ce qui concerne de la production au Sud, le label européen ECOCERT est le plus courant. Il offre certains avantages comparatifs par rapport au label Bourgeon, le plus connu en Suisse. Il n'exige pas la conversion de toute l'exploitation, permet l'usage de l'avion comme moyen d'acheminement et dispose de plusieurs antennes en Afrique, permettant ainsi une réduction des frais de certification et de monitoring.

Les exigences requises pour la certification biologique doivent être complétées par celles du marché. Premièrement, les perspectives de marché ne sont pas identiques pour tous les produits biologiques. Selon les distributeurs, les produits exotiques qui n'ont pas encore atteint le niveau de consommation de masse, comme par exemple certains fruits, ont peu de chance de percer dans le domaine biologique à court terme. L'introduction de la production biologique dans un pays du Sud doit donc être précédée d'une étude de marché détaillée, et ce d'autant plus que la prime «bio» est rarement percevable localement, la demande nationale pour ces produits plus chers étant très faible. De plus, la prime permet parfois juste de compenser la baisse de rendement et le travail supplémentaire, comme c'est le cas actuellement pour le coton bio en Afrique de l'Ouest. Deuxièmement, les exigences concernant par exemple les volumes 
requis et les pratiques commerciales sont identiques à celles du marché «traditionnel». Troisièmement, le consommateur de denrées alimentaires biologiques tend de surcroît à privilégier toujours davantage l'offre locale (CNUCED 2001).

\section{$\square$ Les labels «équitables»}

Le label du commerce équitable est basé non pas sur le mode de production, mais sur la qualité des relations entre le producteur et l'acheteur ainsi que sur le mode de gestion de la coopérative de producteurs. Les organisations de commerce équitable s'engagent à fournir un accès direct aux marchés du Nord pour les produits du Sud en cherchant à minimiser les coûts de transaction et à permettre le paiement d'un prix équitable. Ce prix doit couvrir les besoins de base des producteurs et les coûts de production, et laisser une marge d'investissement. Un autre engagement pris par les organismes envers les producteurs est de permettre un prépaiement et de garantir des relations et des contrats à long terme (EFTA 1998). Les producteurs s'engagent eux à s'organiser en association ou coopérative, gérée de manière démocratique, et à redistribuer les revenus.

Contrairement au label bio, le commerce équitable n'est pas légiféré en Suisse. En conséquence, plusieurs organismes offrent des produits issus du «commerce équitable», avec des exigences, des contrôles et des formes d'appui aux producteurs parfois très différents. Depuis 1999, la majorité des acteurs du commerce équitable en Suisse se sont regroupés dans un Forum suisse pour le commerce équitable, afin d'harmoniser les critères. Schématiquement, deux approches peuvent être distinguées. La première part des conditions-cadres locales et cherche à valoriser les ressources existantes. Cette approche, avec les Magasins du Monde comme pionniers en Suisse, a permis l'implantation de produits issus du commerce équitable et de nouveaux produits exotiques en Suisse. La deuxième approche se base sur la demande du marché et recherche des partenaires producteurs. Max Havelaar en est un exemple. L'approche des organismes de commerce équitable détermine implicitement les critères à remplir par les producteurs et le type de partenariat avec les producteurs au Sud. Schümperli Younossian (2001) montre que Claro S.A., une centrale d'importation de produits commercialisés de manière équitable, fournissant les Magasins du Monde, s'adresse à des producteurs du Sud ayant des expériences et des niveaux de ressources différents. L'accompagnement des petits producteurs pour leur permettre d'atteindre les degrés de compétence requis pour l'exportation est un aspect important de Claro S.A. (Schümperli Younossian 2001). Max Havelaar s'adresse plutôt à des producteurs ayant souvent déjà une expérience d'exportation et capables de fournir de manière régulière des volumes importants et de qualité. Si une association de producteurs n'est pas en mesure de répondre à ces critères élevés, la fondation Max Havelaar l'encouragera à recevoir l'assistance d'une organisation de développement et, le cas échéant, se chargera d'établir le contact. La barrière à la certification existe aussi pour des grands producteurs du secteur plus traditionnel. Par exemple, les bananes Chiquita, malgré l'association de Chiquita avec l'ONG Rainforest Alliance dans le cadre du projet «Better Banana Project» (Gassmann 2001) pour la mise en place d'un nouveau label plus respectueux de l'environnement et plus responsable sur le plan social, ont probablement peu de chance d'obtenir la certification Max Havelaar. 
Cette différenciation des exigences à la production s'explique en grande partie par les circuits de commercialisation des produits. Le consommateur de produits vendus par les Magasins du Monde fait un choix explicite de soutien au commerce équitable en se déplaçant vers ces magasins. La force de Max Havelaar est d'avoir réussi à introduire ses produits certifiés dans les supermarchés traditionnels, facilitant ainsi l'accès de ces produits et permettant des volumes d'écoulement importants. De plus, le surplus de prix à la consommation est peu élevé: 10 centimes par litre de jus d'orange par exemple. Implicitement, les exigences au niveau de la qualité et des quantités sont identiques à celles des produits traditionnels vendus en grandes surfaces. En conséquence, les produits susceptibles d'obtenir le label Max Havelaar sont des produits de consommation de masse et produits par des coopératives. Les distributeurs reconnaissent les avantages à l'introduction de produits issus du commerce équitable dans leur étalage: diversification de l'offre, image sociale, produits de qualité, demande croissante, etc. Toutefois, leur distribution implique des coûts et risques supplémentaires pour l'importateur/distributeur: risque de variation du prix mondial par rapport à un prix d'achat fixe, frais de licence, volumes parfois moins importants que dans le secteur traditionnel. Actuellement, les parts de marché des produits Max Havelaar en Suisse sont de 3,5\% pour le jus d'orange, 5\% pour le café et $15 \%$ pour les bananes, et le chiffre d'affaires en Suisse se montait à 62 millions en 1999 (Max Havelaar 1999). Au niveau européen, le taux de croissance global du commerce équitable est estimé entre 10 et $25 \%$, cela surtout grâce à l'introduction de nouveaux produits sur de nouveaux marchés (Ronchi 2000). Actuellement, ces produits sont peu transformés dans les pays du Sud. Plus de $60 \%$ des 2500 produits qui portent des labels de commerce équitable sont des produits primaires, tels le café, le thé, le miel, le cacao, le sucre et la banane (Ronchi 2000).

\section{$\square$ Les labels $d u$ «Sud»}

Force est de constater que la grande majorité des labels «durables» ont été conçus au Nord et que peu de pays du Sud disposent d'un service de certification public et/ou d'un cadre légal pour légiférer sur les labels. Toutefois, il existe de plus en plus d'initiatives visant à améliorer la valorisation de la production locale.

Le Bénin a développé un label de qualité pour son coton fibre, label largement reconnu sur le marché mondial de la fibre. Il est basé sur les qualités intrinsèques de la fibre comme sa longueur, sa couleur, la présence d'imperfections, etc., intègre la traçabilité jusqu'au village de production et permet le paiement d'une prime (ou d'une réfaction) par rapport à un prix de référence, le prix mondial$^{2}$, aux détenteurs de la fibre, c'est-à-dire aux égreneurs. Les conditions de production ayant aussi une influence sur ces paramètres, le prix au producteur est différencié selon la qualité du coton graine. Toutefois, en raison du système actuel de fixation des prix, un lien direct entre la prime qualité payée à l'exportation et le prix payé au producteur n'existe pas. Un autre exemple est le SQMM (Safe Quality Mango Management), créé par l'association SAMGA (South African Mango Growers' Association), regroupant la quasi-totalité des

2. Indice A de Liverpool. 
producteurs de mangues d'Afrique du Sud. Ce label est essentiellement destiné à faciliter l'accès des fruits sur le marché international ouvert suite à la suppression de l'embargo (Finnemore, communication personnelle). Il inclut le concept HACCP (Hazard Analysis Critical Control Points), exigé par l'Union européenne, et est basé sur le mode de production intégrée. Seuls les producteurs membres accrédités de l'association peuvent utiliser le logo, un autocollant à fixer sur chaque palette de fruits (SAMGA 1999). Un troisième exemple est le label Homegrown développé au Kenya par une entreprise privée pour des produits horticoles. Il est basé sur les critères de production intégrée et inclut des aspects sociaux (Evans 1999). La force de cette entreprise est d'avoir réussi à «remonter» la chaîne jusqu'en Europe grâce à son label. Elle livre directement aux supermarchés des légumes préemballés. $90 \%$ de sa production sont cultivés sur ses terres, le solde étant produit par de petits exploitants travaillant en soustraitance. Ces exemples sont assez représentatifs du fait que les initiatives locales portent essentiellement sur des produits d'exportation comme les produits traditionnels, cacao, thé, coton, et plus récemment les produits horticoles. La principale raison est le coût de certification et de mise en place d'organismes de certification.

\section{MESURES POUR ACCROÎTRE LES EXPORTATIONS DES PAYS DU SUD}

Pour la majorité des pays d'Afrique subsaharienne, le produit national brut par habitant est inférieur à 700 USD, soit environ 55 fois plus petit que celui de la Suisse (World Bank 2001). Une diversification et une plus grande valorisation de la commercialisation sur les marchés local et international peuvent contribuer à augmenter leur revenu, voire à réduire ces disparités. Il existe plusieurs pistes pour améliorer l'accès des producteurs des pays du Sud aux marchés et pour tirer parti des possibilités de commercialisation, voire d'exportation.

Des politiques cohérentes, aux niveaux local et international, sont indispensables pour permettre un meilleur accès des produits des pays du Sud aux marchés régional et international et un rééquilibrage du niveau de mise en valeur des produits entre le Nord et le Sud. Plusieurs mesures sont à prévoir. La première est une harmonisation des politiques de soutien à la production et à la commercialisation, et ce autant au niveau régional pour le commerce Sud-Sud qu'au niveau international. Il en va de même pour les prescriptions des autorités réglementaires nationales. Les calculs des coûts à la production doivent être revus et harmonisés entre le Nord et le Sud. Les coûts non monétaires, comme le temps pour la formation et/ou le conseil et le paiement de la main-d'œuvre en nature (par exemple repas), sont nombreux et peuvent représenter une part importante des coûts totaux (Crole-Rees et Soulé 2001). Ils doivent être inclus dans les coûts pour une plus grande équité entre le Nord et le Sud. Les aspects sociaux et environnementaux doivent aussi apparaître dans les coûts, au Sud comme au Nord.

Les goûts et préoccupations des consommateurs varient entre autres selon les régions, les périodes et les classes de revenus. Les opportunités de cette demande différenciée peuvent être exploitées par la différenciation des produits. Les labels peuvent aussi être utilisés à cet effet, pour autant que l'information 
soit claire et juste. Cela est une des raisons principales pour la coexistence entre les labels publics et privés dans le futur. Les labels privés permettent d'exploiter les avantages comparatifs, particulièrement des entreprises importantes, alors que les labels publics, moins exigeants, permettent d'inclure la majorité des producteurs (Reardon et al. 2001). Les coûts de certification étant élevés, particulièrement pour les petits producteurs, il est important de permettre de les inclure en finançant certaines activités «non rentables» (Schümperli Younossian 2001). Les promoteurs de produits labellisés devraient prévoir des stratégies visant non seulement la production et la commercialisation de produits sous label, mais aussi une diversification des produits et leur mise en valeur, par la transformation et l'emballage, au niveau local.

Pour s'approprier une partie au moins de la valeur ajoutée considérable créée aux stades de la commercialisation et de la distribution, les exportateurs des pays du Sud devraient privilégier des spécialités et autres produits de créneau et élargir leur offre. On peut citer des produits de contre-saison qui ont aussi l'avantage de ne pas concurrencer l'offre locale du Nord. Augmenter la transformation et l'emballage dans le pays producteur peut accroître la marge de manière substantielle. Les opérations à mettre en place peuvent être relativement simples, comme le séchage par exemple, ou plus élaborées, comme la fabrication de jus ou de salade de fruits frais. A ce titre, il est impératif de mettre sur pied des stratégies spécifiques au niveau de l'entreprise. Ainsi, au Burkina Faso, les sécheurs de mangues commercialisent la première qualité en Europe et la deuxième localement. Les ressources locales doivent aussi être mieux valorisée, localement comme sur les marchés régionaux et internationaux. Beaucoup d'opportunités se présentent pour des marchés à remplir avec des fruits, épices, herbes médicinales, etc. Cette approche, dite de «marché de niche», est particulièrement adaptée aux petites exploitations (Ramírez Farías 2001). De plus, elle permet de maintenir et de valoriser la biodiversité, comme par exemple l'initiative de TechnoServe, en collaboration avec une station de recherche, des exportateurs et des producteurs, en vue d'améliorer la qualité de production et la commercialisation d'une variété de pois. Le marché visé est le marché de niche à haute valeur ajoutée (pois frais et transformé) en Inde et en Europe (TechnoServe, communication personnelle).

Pour toute initiative, le partenariat à moyen et à long terme est à privilégier. Barjolle (2001) a montré que certains types d'associations peuvent contribuer à réduire les coûts de transaction. De nature commerciale ou mutuelle, formelle ou informelle, les partenariats permettent en général un meilleur accès à l'information et à la formation. Le partenariat entre producteurs et importateurs, comme c'est le cas avec le label Max Havelaar, permet un débouché garanti sur le moyen terme. Les importateurs de fruits exotiques et les commerçants de coton d'Afrique de l'Ouest, en règle générale spécialisés dans ces branches, travaillent pour la plupart sur la base d'un partenariat à moyen terme avec différents groupes de producteurs. Des arrangements peuvent donc être mis en place, tels que prépaiement, contrat sur plusieurs années et fixation d'un prix de base. $\mathrm{Au}$ niveau de la production, les producteurs de mangues d'Afrique du Sud se sont mis en contact avec les producteurs de Guinée, avec pour objectif d'élargir la période de récolte, diversifier leur production et renforcer le savoir-faire des deux pays. Au Kenya, un partenariat tripartite a permit à Homegrown de devenir un des leaders africains pour l'exportation de légumes frais préemballés vers 
les supermarchés d'Angleterre. Ce partenariat comprend le gouvernement et des institutions internationales de développement. Des interprofessions, incluant producteurs et transformateurs, se mettent en place, comme pour l'arachide au Sénégal et le coton au Bénin. De plus en plus, certains pays industrialisés se mettent aussi à rechercher des solutions à leurs problèmes de qualité avec l'aide du Sud et instaurent des collaborations. Les producteurs de mangues de Floride se sont par exemple mis en contact avec ceux d'Egypte pour améliorer leur pool végétal avec le soutien d'un organisme de développement (International Focus 2000).

La commercialisation et plus particulièrement l'exportation exigent de plus en plus de professionnalisme et d'esprit d'entreprise. Des études récentes ont montré que les exportateurs sont plus enclins à prendre des risques que les entrepreneurs souhaitant exporter (Streeter et Bills 1998), que la taille des entreprises a une influence sur leur capacité à obtenir l'information (Ades et Glaeser 1999) et à s'ajuster pour l'obtention des labels (Reardon et al. 2001). La formation à l'entrepreneuriat doit tenir compte de ces éléments. Concrètement, cela implique une flexibilité et une adaptation de la durée de la formation au public cible.

Au niveau de la recherche, on veillera à réduire la disparité des moyens investis dans la recherche entre les filières traditionnelles et non traditionnelles. La recherche devrait se pencher d'une part sur la production et la transformation des ressources locales pour le marché local comme le marché international, et d'autre part sur les produits non traditionnels et autres produits de niche qui ont des chances de succès sur les marchés régionaux du Sud et sur ceux du Nord. Des études pourraient aussi être réalisées sur l'utilisation des noms de marque et des appellations d'origine contrôlée par exemple.

Les flux d'information, en tant qu'outil stratégique pour tous les acteurs de la chaîne alimentaire, doivent être intensifiés afin de pallier la distance grandissante, au propre comme au figuré, entre le producteur et le consommateur. Pour les producteurs, les informations doivent comprendre les prix et coûts tout comme les différentes possibilités de commercialisation, les méthodes de paiement, etc. L'initiative des systèmes d'information des marchés en Afrique de l'Ouest, qui diffuse son information chaque semaine sous forme de bulletin et par le biais des radios rurales, est un exemple de la promotion des services d'information.

\section{CONCLUSION}

Il est souvent maintenu que les agriculteurs et les distributeurs des pays du Sud comme du Nord, bien que sensibles aux aspects sociaux et environnementaux, continuent à se soucier davantage d'améliorer leur revenu à court terme que de préserver l'environnement à plus long terme. Du côté des consommateurs, des suggestions ont été faites pour une «alimentation efficace» qui favorisent l'agriculture biologique, indigène et de plein air et les produits équitables (WWF 2000) alors que l'on reproche souvent au consommateur de rechercher son plaisir à court terme et à moindre prix. Chère consommatrice, cher consommateur, qu'y a-t-il eu dans votre dernière assiette? 


\section{RÉFÉRENCES}

Ades A. et Glaeser E., 1999, «Evidence on Growth, Increasing Returns and the Size of the Market», Quaterly Journal of Economics, vol. 3, n 114, pp. 1025-1046.

Auroi C. et Schümperli Younossian C. (dir.), 2001, Le commerce durable. Vers de plus justes pratiques commerciales entre le Nord et le Sud, Genève: IUED.

Barjolle D., 2001, Hybrid Forms of Governance: The Case of Artisanal Food Products, Contribution préparée pour le $78^{\mathrm{e}}$ séminaire EAAE, Danemark, juillet.

CBI et SIPPO (Swiss Import Promotion Programme), 1999, Exporting to Switzerland and the European Union, Rotterdam and Zurich: SIPPO.

CNUCED, 2001, Moyens d'accroître la capacité des pays en développement de produire et d'exporter des produits agricoles et alimentaires, y compris des biens très spécialisés, et notamment des produits écologiques, Note d'information établie par le secrétariat de la CNUCED, TD/B/COM.1/EM. $15 / 2$, Genève, 11 mai.

Crole-Rees A. et Soulé B.G., 2001, Etude relative au mécanisme de formation des prix de cession de coton graine et des intrants agricoles aи Bénin, Rapport final, vol. 1., Lausanne et Cotonou, octobre.

Damary P., 2001, «Le label de qualité en agriculture biologique: un pionnier dans le domaine du commerce durable», in Auroi C. et Schümperli Younossian C. (2001), pp. 137-152.

Diopp S.G., 1999, Conditions for Food Manufactoring in Sub-Saharan Africa, Contribution to the Workshop on Food Chain in Sub-Saharan Africa held in Bamako, CASIN (Centre for Applied Studies in International Negociations), SG2000, 15-19 October.

Evans R.K., 1999, «From Small Farms to Supermarkets», in Breth S.A. (ed.), Partnerships for Rural Development in Sub-Saharan Africa, CASIN/SAA/SGGlobal 2000, Geneva.

EFTA (European Fair Trade Association), 1998, Commerce équitable. Mémento pour l'an 2000, Maastricht: EFTA.

FiBL (Forschungsinstitut für biologischen Landbau) et SIPPO (Swiss Import Promotion Programme), 2001, The Organic Market and the European Union: Overview and Market Access Information for Producers and International Trading Companies, January.

FIDA (Fonds international de développement agricole), 2001, Rapport 2001 sur la pauvreté rurale: comment mettre fin à la pauvreté rurale, Rome: FIDA, septembre.

Gassmann R., 2001, «Chiquita, entreprise responsable», Construire, n 14, 3 avril.

International Focus, 2000, «UF Seeks Mango Exchange with Egypt to Improve Production », International FOCUS, vol. 11, n ${ }^{\circ}$, March-April.

Lhéraut G., 2001, Logistique d'exportation des pays ACP pour les fruits et légumes et les produits horticoles, Contribution préparée pour le séminaire africain francophone sur la diversification et le développement du secteur horticole, Bamako, 13-15 février.

Max Havelaar, 1999, Fondation Max Havelaar. Rapport annuel, Bâle.

Rabobank International, 2001, Fresh Supplies for Asia, Asia Fruit Congress 2001, 9 May.

Ramírez Farías L., 2001, «Globalisation and Livelihood Diversification through Non-traditional Agricultural Products: The Mexico Case», ODI Natural Resource Perspectives, n 67, June.

Reardon T., Codron J.-M., Busch L., Bingen J. et Harris C., 2001, «Global Change in Agrifood Grades and Standards: Agribusiness Strategic Responses in Developing Countries », International Food and Agribusiness Management Review, vol. 2, n 3-4, pp. 421-435.

Roddick A., 1991, Body and Soul, London: Vermillon.

Ronchi L., 2000, Fair Trade in Costa Rica: An Impact Report, Brighton: University of Sussex.

SAMGA (South African Mango Growers' Association), 1999, Safe Quality Mango Management (SQMM) : Protocol, Tzaneen.

Schümperli Younossian C., 2001, «Pourquoi subventionner le commerce équitable», in Auroi C. et Schümperli Younossian C. (2001), pp. 227-249.

Streeter D. et Bills N., 1998, «Information Strategies for Agricultural Exporters», International Food and Agribusiness Management Review, vol. 1, n 1, pp. 25-40.

World Bank, 2001, World Development Report 2000/2001 : Attacking Poverty, Washington, D.C.: World Bank.

WWF, 2000, Le guide des labels, Vernier: WWF Suisse. 